\title{
A differential-delay estimator for thermoacoustic oscillations in a Rijke tube using in-domain pressure measurements*
}

\author{
Jean Auriol ${ }^{1}$, Gustavo Artur de Andrade ${ }^{2}$, Rafael Vazquez ${ }^{3}$
}

\begin{abstract}
In this work, we study the observer design problem for estimating thermoacoustic instabilities in a Rijke tube, using an in-domain point pressure measurement. Writing the system model in Riemann coordinates and, after a "folding transformation", it takes the form of $4 \times 4$ linear hyperbolic partial differential equations (PDEs) coupled at the boundaries with a linear ordinary differential equation (ODE). This results in a PDE-ODE observer design problem, whose output is a combination of two infinite-dimensional states measured inside the domain. As a first step in our observer design, a second "folding" transformation is applied around the measurement point, resulting in a $6 \times 6$ PDE-ODE system with measurements at one boundary. Then, the observer is designed as a copy of these equations plus an output injection term, which is given by a linear operator in the right-hand side of the ODE part. This operator is then chosen so that convergence of estimates is guaranteed by using stability properties of differentialdelay systems. The design extends a previous result, based on backstepping, that required two measurements (both pressure and velocity). Simulation results are presented to illustrate the effectiveness of the proposed observer design.
\end{abstract}

\section{INTRODUCTION}

The Rijke tube is the simplest device that produces thermoacoustic instabilities [19]. It was discovered in the 1850s by P. L. Rijke [15] and consists of a vertical tube open at both ends with an embedded heat source. The air that traverses the heating zone expands, causing the local pressure to increase. The pressure acoustically propagates along the tube and returns (due to the boundary conditions). This behavior induces a feedback loop: the pressure at the current time is affected by itself at earlier time instants, leading to time-delayed dynamics in the thermoacoustic coupling. Thus, a hyperbolic partial differential equation (PDE) system for the acoustic dynamics plus an ordinary differential equation (ODE) describing the heat release dynamics is suitable to mathematically represent this system [6].

Traditionally, the Rijke tube has been used as a benchmark system to study thermoacoustic instabilities. These are present in many engineering applications, such as blast furnaces, heating units, gas turbines or rocket engines, all of them sharing the common point of displaying instabilities that are, in general, unexpected, undesirable and often of significant amplitude. At best, they produce vibrations potentially affecting delicate instrumentation and payloads. At their worst, the oscillations may increase the average pressure, resulting even in rupture of the system. One often

\footnotetext{
*The authors gratefully acknowledge financial support from Fundación Carolina, and from the Spanish Ministerio de Ciencia, Innovación y Universidades under grant PGC2018-100680-B-C21. ${ }^{1}$ Jean Auriol is with Université Paris-Saclay, CNRS, CentraleSupélec, Laboratoire des signaux et systèmes, 91190, Gif-sur-Yvette, France. jean.aurioldcentralesupelec. fr ${ }^{2}$ Gustavo Artur de Andrade is with Department of Automation and Systems, Universidade Federal de Santa Catarina, 88040-900, Florianópolis, SC, Brazil. gustavo.arturdufsc.br ${ }^{3}$ Rafael Vazquez is with Department of Aerospace Engineering, Universidad de Sevilla, Camino de los Descubrimientos, s.n.,41092 Sevilla, Spain rvazquez1@us.es
}

cited example of these instabilities is the F-1 engine, that was designed in the 1950s to power the Saturn V rocket [10]. The pressure oscillations encountered in this engine had amplitudes of more than 2000 psi and the modelling challenges presented by this phenomenon during the design stage resulted in long and costly testing campaigns.

These problems motivate the need to understand the underpinnings of thermoacoustic instabilities and to develop feedback control systems that mitigate the resulting oscillations. Most control laws found in the literature use modal decomposition as proposed by [13], which employs a Galerkin method. Generally, only the first mode is unstable in open-loop thermoacoustic systems and thus reduced models typically only considers this unstable portion. A detailed review of the main control strategies considered during the past fifty years can be read in [19]. Methods include phaseshifting [18], [7], linear quadratic gaussian controllers [8], model-based predictive control [11] and sliding mode control [16], [9], among others.

However, as shown in [14], control strategies based on modal decomposition can destabilize higher modes ${ }^{1}$ that were neglected in the design stage. In this context, control systems based on infinite dimensional dynamics-without any model reduction-may be more reliable and have better performance in practice. Recent approaches along this line can be seen in [5], [17] and references therein.

From a practical point of view, state observers are crucial tools for control systems based on infinite dimensional models, since in general the available sensors are not able to fully measure the entire spatial domain, which are often required in the resulting feedback laws. In the context of estimation of thermoacoustic instabilities in Rijke tubes, a backstepping design was proposed in [4] considering the linearized PDE-ODE model and downstream boundary pressure measurements. In [2], a state observer based on the linearized PDE and the nonlinear ODE model was developed assuming upstream boundary pressure measurements (at one of the open ends of the tube). These designs employed a Riemann coordinates transformation and the idea of folding the spatial domain around the ODE coupling point, in order to map the system into transport equations and to displace the coupling between the PDEs and the ODE to the boundary of this new equivalent system.

However, boundary pressure measurements at the end of the tube are typically problematic in systems involving thermoacoustic instabilities, because at those points there are pressure nodes which induce poor measurements [19]. To tackle this issue, a state observer based on in-domain pressure and velocity measurements was proposed in [3] extending the backstepping boundary observer of [4]. The same idea of Riemann coordinates transformation and folding was

\footnotetext{
${ }^{1}$ This phenomenon is also referred to as control spillover.
} 
applied in that work, but a second folding is done at the in-domain measurement point in order to translate it to the boundary.

We start by applying the same folding transformation on the linearized PDE-ODE Rijke tube model, however, since pressure is much easier to measure than velocity, in this work we design an observer relying only on in-domain pressure measurements (that is, one measurement instead of two). The challenge of this observer design is that the available measurement is a linear combination of two states at one boundary when written in Riemann coordinates. We design the observer as a copy of the system's equations plus an output injection term, which is given by a linear operator, in the right-hand side of the ODE. Using the method of characteristics and the theory of delayed differential equations, the linear operator is designed such that the exponential convergence of the error dynamics in the $L^{2}$-norm is guaranteed. Simulation results are presented to illustrate the effectiveness of the proposed observer design.

The rest of the paper is organized as follows. Some results on time-delay systems that are used on the paper are presented in Section II. The system description and the observer problem statement are given in Section III. The observer design and the proof of its convergence are detailed in Section IV. Simulation results for the proposed observer are given in Section V. We finish with some concluding remarks and suggestions for future works in Section VI.

\section{SOME PRELIMINARY RESULTS ON TIME-DELAY SYSTEMS}

In this section we recall an important result on scalar timedelay systems, which is crucial in the design of the state observer.

Let us denote by $L^{2}\left(\left[-\tau_{1}, 0\right], \mathbb{R}\right)$ the Banach space of $L^{2}$ functions mapping the interval $\left[-\tau_{1}, 0\right]$ into $\mathbb{R}$, where $\tau_{1}$ is a positive delay. Consider the following system

$$
\dot{z}(t)=-a z(t)-b z\left(t-\tau_{0}\right),
$$

where $z$ belongs to $L^{2}\left(\left[-\tau_{0}, 0\right], \mathbb{R}\right), a$ and $b$ are two real constant, $\tau_{0}$ is a positive delay. We have the following stability theorem, which is directly adjusted from [12, Proposition 3.15].

Theorem 1: The following assertions hold:

1) The system (1) is asymptotically stable if $a+b>0$ and $a \geq|b|$.

2) The system (1) is asymptotically stable if $b>|a|$ and $\tau_{0}<\tau^{\star}$ where $\tau^{\star}$ is obtained from

$$
\tau^{\star}=\frac{\arccos \left(-\frac{a}{b}\right)}{\sqrt{b^{2}-a^{2}}} .
$$

This theorem means that, under some conditions on the coefficients $a$ and $b$ and on the delay $\tau_{0}$, the system (1) is naturally asymptotically stable.

\section{SYSTEM UNDER CONSIDERATION}

In this section, we describe the model we use for the Rijke tube (see Figure 1). This model is inspired by the one described in [6], and it is given by the following set of linear PDEs:

$$
\begin{aligned}
\partial_{t} v(t, x)+\frac{1}{\bar{\rho}} \partial_{x} P(t, x) & =0, \\
\partial_{t} P(t, x)+\gamma \bar{P} \partial_{x} v(t, x) & =\frac{\bar{\gamma}}{A} \delta\left(x-x_{0}\right) Q(t),
\end{aligned}
$$

$$
\tau_{h r} \dot{Q}(t)+Q(t)=f^{\prime}(\bar{v})\left(T_{w}-\bar{T}_{\text {gas }}\right) v\left(t, x_{0}\right),
$$

where the time $t[\mathrm{~s}]$ belongs to $[0, \infty)$, the space variable $x[\mathrm{~m}]$ belongs to $[0, L], L$ being the length of the tube. We have denoted by $\delta$ the Dirac delta distribution $\left[\mathrm{m}^{-1}\right]$. The different states are the velocity fluctuations $v\left[\mathrm{~m} \mathrm{~s}^{-1}\right]$, the pressure fluctuations $P[\mathrm{~Pa}]$, and the heat power release $Q$ [W]. We have denoted by $\bar{v}$ the steady-state velocity [m $\left.\mathrm{s}^{-1}\right]$, by $\bar{\rho}$ the steady-state density $\left[\mathrm{kg} \mathrm{m}^{-3}\right]$ and by $\bar{P}$ the steady-state pressure $[\mathrm{Pa}]$. The tube cross-section area $\left[\mathrm{m}^{2}\right]$ is given by $A$, while the heat capacity ratio is given by $\gamma=\frac{C_{P}}{C_{V}}\left(C_{P}\right.$ and $C_{V}$ being the specific heat capacity $\left[\begin{array}{ll}\mathrm{J} \mathrm{kg}^{-1} & \mathrm{~K}^{-1}\end{array}\right]$ at constant pressure and volume conditions, respectively). The location of the heat release source [m] is given by $x_{0} \in(0, L)$, while the heat release time constant [s] is denoted $\tau_{h r}>0$. The heat power transfer $[\mathrm{W} / \mathrm{K}]$ is given by $f(\bar{v})=l_{w}\left(\kappa+\kappa_{v} \sqrt{|\bar{v}|}\right)>0$ with $\kappa$ being the fluid thermal conductivity $\left[\mathrm{W} \mathrm{m} \mathrm{m}^{-1} \mathrm{~K}^{-1}\right], l_{w}$ the wire length $[\mathrm{m}], \kappa_{v}$ an empirical constant $\left[\mathrm{W} \mathrm{s} \mathrm{s}^{1 / 2} \mathrm{~m}^{-1 / 2} \mathrm{~K}^{-1}\right]$ and $\bar{\gamma}=\gamma-1>0$. Finally, we have denoted by $T_{w}$ and $\bar{T}_{g a s}$ the wire and gas temperature $[\mathrm{K}]$, which verify $T_{w}-\bar{T}_{g a s}>0$. The system (2)-(4) has the following boundary conditions

$$
P(t, 0)=-Z_{0} v(t, 0)+U(t), P(t, L)=Z_{L} v(t, L),
$$

where $Z_{L}$ and $Z_{0}$ are (non-zero) reflection losses $\left[\mathrm{kg} \mathrm{m}^{-2}\right.$ $\left.\mathrm{s}^{-1}\right]$, and $U$ is the control input $[\mathrm{Pa}]$. The initial conditions of the system are denoted $v_{0}, P_{0}$ and $Q_{0}$. They belong to $L^{2}([0, L]) \times L^{2}([0, L]) \times \mathbb{R}$.

\section{A. Observer problem}

The objective of this paper is to design a state observer that only relies on the measurement of the pressure fluctuations at some arbitrary location $x_{m}[\mathrm{~m}]$. More precisely, we want to estimate the states $Q, P$ and $v$, only using the knowledge of $U(t)$ (input) and of

$$
y(t)=P\left(t, x_{m}\right),
$$

with $x_{m} \in(0, L) \backslash\left\{x_{0}\right\}$. This problem is more general than the one considered in [3] where $x_{m}$ was assumed to be equal to $L$ and to the one tackled in [4], where a second measurement (namely $v\left(t, x_{m}\right)$ ) was also necessary. Note that the point $x_{m}$ can be chosen to avoid pressure nodes. In what follows, we assume that $x_{m}>x_{0}$. However, the method we consider here can easily be extended to the case $x_{m}<x_{0}$.

\section{B. Characteristic coordinates and folding transformation}

In this section we will rewrite (2)-(4) as a system with a diagonalized transport matrix (i.e. a system composed of transport equations with source terms couplings) and remove the Dirac delta from the right-hand side. The computations were based on [5], [3] and only the main steps will be presented here. The interested reader is refereed to [3], [5] for more details. Finally, it is important to emphasize that the computations are assuming that $x_{m}>x_{0}$, but the method can easily be extended to the case $x_{m}<x_{0}$. Consider the following Riemann invariants:

$$
\begin{aligned}
& P(t, x)=\frac{1}{2}\left(R_{1}(t, x)+R_{2}(t, x)\right), \\
& v(t, x)=\frac{1}{2 \sqrt{\gamma \bar{P} \bar{\rho}}}\left(R_{1}(t, x)-R_{2}(t, x)\right) .
\end{aligned}
$$




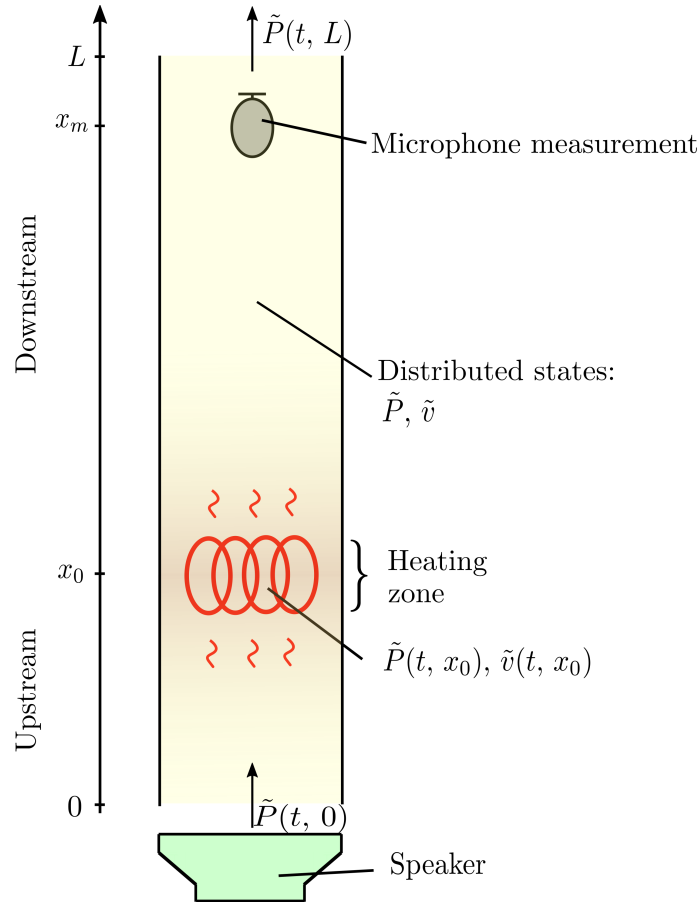

Fig. 1. A diagram depicting the Rijke tube and its main components.

Moreover, to remove the Dirac delta distribution term and to translate the measurements to the boundary conditions, we consider the folding transformation given by

$$
\begin{aligned}
& R_{1}(t, x)= \begin{cases}\alpha_{1}(t, x), & x \in\left[0, x_{0}\right], \\
\beta_{2}(t, x), & x \in\left[x_{0}, x_{m}\right], \\
\alpha_{3}(t, x), & x \in\left[x_{m}, L\right],\end{cases} \\
& R_{2}(t, x)= \begin{cases}\beta_{1}(t, x), & x \in\left[0, x_{0}\right], \\
\alpha_{2}(t, x), & x \in\left[x_{0}, x_{m}\right], \\
\beta_{3}(t, x), & x \in\left[x_{m}, L\right],\end{cases}
\end{aligned}
$$

and define the piecewise spatial transformation in $z$

$$
z= \begin{cases}\frac{x}{x_{0}}, & x \in\left[0, x_{0}\right], \\ \frac{L-x}{L-x_{0}}, & x \in\left[x_{0}, x_{m}\right], \\ \frac{x-x_{m}}{L-x_{m}}, & x \in\left[x_{m}, L\right],\end{cases}
$$

Combining the Riemann invariants and the folding transformation, the original system (2)-(4) can be rewritten as

$$
\begin{aligned}
\partial_{t} \alpha(t, z)+\Lambda \partial_{z} \alpha(t, z) & =0, \\
\partial_{t} \beta(t, z)-\Lambda \partial_{z} \beta(t, z) & =0, \\
\tau_{h r} \dot{Q}(t)+Q(t) & =c_{2}\left(\alpha_{1}(t, 1)-\alpha_{2}(t, 1)\right),
\end{aligned}
$$

where

$\alpha=\left[\alpha_{1}, \alpha_{2}, \alpha_{3}\right]^{T}, \beta=\left[\beta_{1}, \beta_{2}, \beta_{3}\right]^{T}, \Lambda=\operatorname{diag}\left\{\lambda_{1}, \lambda_{2}, \lambda_{3}\right\}$. The boundary conditions are given by

$$
\begin{gathered}
\alpha(t, 0)=N_{i} \beta(t, 0)+N_{u} U(t), \\
\beta(t, 1)=N_{f} \alpha(t, 1)+N_{q} Q(t),
\end{gathered}
$$

where

$$
N_{i}=\left(\begin{array}{ccc}
k_{0} & 0 & 0 \\
0 & 0 & 1 \\
0 & 1 & 0
\end{array}\right), \quad N_{u}=\left(\begin{array}{l}
2 \\
0 \\
0
\end{array}\right)
$$

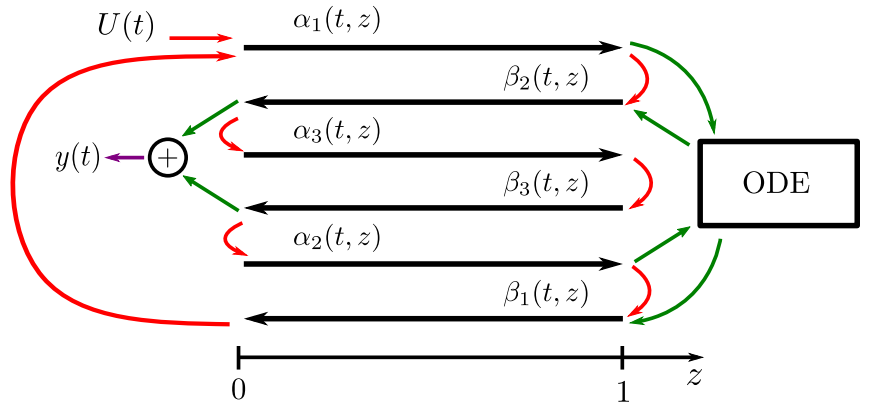

Fig. 2. Schematic representation of the interconnected system (12)-(16).

$$
N_{f}=\left(\begin{array}{ccc}
0 & 1 & 0 \\
1 & 0 & 0 \\
0 & 0 & k_{L}
\end{array}\right), \quad N_{q}=\left(\begin{array}{c}
c_{1} \\
c_{1} \\
0
\end{array}\right)
$$

The different constant are defined by $c=\sqrt{\gamma \frac{\bar{P}}{\bar{\rho}}}$ (speed of sound), $c_{1}=\frac{\bar{\gamma}}{A c}, c_{2}=\frac{f^{\prime}(\bar{v})\left(T_{w}-T_{\text {gas }}\right)}{2 \sqrt{\gamma \bar{P} \bar{\rho}}}>0, k_{L}=\frac{Z_{L}-\bar{\rho} c}{Z_{L}+\bar{\rho} c}$ and $k_{0}=\frac{Z_{0}-\bar{\rho} c}{Z_{L}+\bar{\rho} c}$. The transport speeds $\lambda_{1}, \lambda_{2}$ and $\lambda_{3}$ are given by $\lambda_{1}=\frac{c}{x_{0}}, \lambda_{2}=\frac{c}{x_{m}-x_{0}}, \lambda_{3}=\frac{c}{L-x_{m}}$. Finally, the measurement $y(t)$ now verifies

$$
\begin{aligned}
y(t) & =\frac{1}{2}\left(R_{1}\left(t, x_{m}\right)+R_{2}\left(t, x_{m}\right)\right) \\
& =\frac{1}{2}\left(\beta_{2}(t, 0)+\beta_{3}(t, 0)\right) .
\end{aligned}
$$

The system (12)-(16) is schematically pictured in Figure 2. We will define the total transport time as

$$
\tau=\frac{2}{\lambda_{1}}+\frac{2}{\lambda_{2}}+\frac{2}{\lambda_{3}} .
$$

\section{Physical properties}

In this work, we assume that the physical parameters of the system (12)-(16) satisfies the two following conditions:

Condition 1: The coefficients $k_{0}$ and $k_{L}$ verify $\left|k_{0}\right|<$ $1,\left|k_{L}\right|<1$.

Condition 2: The coefficients of the system verify the following inequalities:

$$
\begin{aligned}
c_{2} c_{1} k_{0} & <-1, \\
\frac{2}{\lambda_{1}} & <\tau_{h r} \frac{\arccos \left(\frac{1}{c_{1} c_{2} k_{0}}\right)}{\sqrt{\left(c_{1} c_{2} k_{0}\right)^{2}-1}},
\end{aligned}
$$

Condition 1 means that the system (12)-(16) would be exponentially stable in the absence of the ODE. Note that this condition is always satisfied in the Rijke tube, as the system always experiences reflection losses in the boundaries. From a mathematical point of view, this condition is necessary to guarantee the robustness of the observer with respect to small delays in the measurements [1]. It means that the operators $\left(1-k_{0} \mathrm{e}^{-\eta s}\right),\left(1-k_{L} \mathrm{e}^{-\eta s}\right)$ and $\left(1-k_{L} k_{0} \mathrm{e}^{-\eta s}\right)$, where $s$ represents the Laplace variable and where $\eta>0$, do not have right-half-plane zeros, and that the corresponding dynamical system is asymptotically stable.

Regarding Condition 2, the inequality (19a) is typically satisfied because the parameter $k_{0}$ assumes values in the interval $(-1,-0.9)$ in most of the prototypes, and the system is constructed with tubes with only a few centimetres of cross-sectional area and lengths around 1 meter, resulting in $c_{1} c_{2}>2$. 
On the other hand, the inequality (19b) is satisfied when the heat addition to the system is sufficiently small. Indeed, since $1 \leq \arccos \left(\frac{1}{c_{1} c_{2} k_{0}}\right)$ it follows that if $2 / \lambda_{1}<$ $\tau_{h r} / \sqrt{\left(c_{1} c_{2} k_{0}\right)^{2}-1}$ holds, then so is (19b). However, note that the denominator of $\tau_{h r} / \sqrt{\left(c_{1} c_{2} k_{0}\right)^{2}-1}$ explicitly depends on the heat power transfer in the system, so the bigger the value of the heat transfer the lower the value of $\tau_{h r} / \sqrt{\left(c_{1} c_{2} k_{0}\right)^{2}-1}$.

As we shall see in the next sections, Condition 2 will be used to prove the convergence of the observer estimates. Although it is not necessary, it considerably simplifies the design.

\section{OBSERVER DESIGN}

The objective of this paper is to design a state-observer that estimates the PDEs and ODE states in (12)-(16) and consequently $Q, v$ and $P$ in (2)-(4). The approach we consider consists on using the transport structure of the error system to rewrite the different states as the solutions of delay equations of neutral type.

In the sequel, we write variables with the superscript ? when referring to estimated states and with the superscript * when referring to error states (difference between estimated and real values).

\section{A. Observer equations}

We design the observer as a copy of the original dynamics with an output injection gain. More precisely, the observer equations read as follows:

$$
\begin{aligned}
\partial_{t} \hat{\alpha}(t, z)+\Lambda \partial_{z} \hat{\alpha}(t, z)=0 & \\
\partial_{t} \hat{\beta}(t, z)-\Lambda \partial_{z} \hat{\beta}(t, z)=0 & \\
\tau_{h r} \dot{\hat{Q}}(t)+\hat{Q}(t)= & c_{2}\left(\hat{\alpha}_{1}(t, 1)-\hat{\alpha}_{2}(t, 1)\right) \\
& -P_{0}(2(y(t)-\hat{y}(t))),
\end{aligned}
$$

where we have denoted $\hat{y}(t)=\frac{1}{2}\left(\hat{\beta}_{2}(t, 0)+\hat{\beta}_{3}(t, 0)\right)$ and where the boundary conditions are given by

$$
\begin{aligned}
& \hat{\alpha}(t, 0)=N_{i} \hat{\beta}(t, 0)+N_{u} U(t), \\
& \hat{\beta}(t, 1)=N_{f} \hat{\alpha}(t, 1)+N_{q} \hat{Q}(t),
\end{aligned}
$$

The operator $P_{0}$ is a linear operator acting on $\tilde{y}(t)=y(t)-$ $\hat{y}(t)$ that still has to be designed. The error system results in

$$
\begin{aligned}
\partial_{t} \tilde{\alpha}(t, z)+\Lambda \partial_{z} \tilde{\alpha}(t, z) & =0 \\
\partial_{t} \tilde{\beta}(t, z)-\Lambda \partial_{z} \tilde{\beta}(t, z) & =0, \\
\tau_{h r} \dot{\tilde{Q}}(t)+\tilde{Q}(t) & =c_{2}\left(\tilde{\alpha}_{1}(t, 1)-\tilde{\alpha}_{2}(t, 1)\right) \\
& +2 P_{0}(\tilde{y}(t)),
\end{aligned}
$$

where the boundary conditions are given by

$$
\begin{aligned}
\tilde{\alpha}(t, 0) & =N_{i} \tilde{\beta}(t, 0), \\
\tilde{\beta}(t, 1) & =N_{f} \tilde{\alpha}(t, 1)+N_{q} \tilde{Q}(t),
\end{aligned}
$$

\section{B. Time-delay representation}

In this section, we rewrite the error system (25)-(29) in a time-delay framework. In particular, we will show that the convergence of $\tilde{Q}$ to zero guarantees the convergence of the whole error system to the origin. Using the method of characteristics and the transport structure of (25)-(29), we obtain $\tilde{\alpha}_{i}(t, 1)=\tilde{\alpha}_{i}\left(t-\lambda_{i}^{-1}, 0\right)$ and $\tilde{\beta}_{i}(t, 0)=\tilde{\beta}_{i}\left(t-\lambda_{i}^{-1}, 1\right)$. Using the boundary conditions:

$$
\begin{aligned}
2 \tilde{y}(t)= & \tilde{\beta}_{2}(t, 0)+k_{L} \tilde{\beta}_{2}\left(t-\frac{2}{\lambda_{3}}, 0\right) \\
\tilde{\beta}_{2}(t, 0)= & k_{0} k_{L} \tilde{\beta}_{2}(t-\tau, 0)+c_{1}\left(\tilde{Q}\left(t-\frac{1}{\lambda_{2}}\right)\right. \\
& \left.+k_{0} \tilde{Q}\left(t-\frac{1}{\lambda_{2}}-\frac{2}{\lambda_{1}}\right)\right) \\
\tilde{\alpha}_{1}(t, 1)= & k_{0} k_{L} \tilde{\alpha}_{1}(t-\tau, 1)+k_{0} c_{1}\left(\tilde{Q}\left(t-\frac{2}{\lambda_{1}}\right)\right. \\
& \left.+k_{L} \tilde{Q}(t-\tau)\right), \\
\tilde{\alpha}_{2}(t, 1)= & k_{0} k_{L} \tilde{\alpha}_{2}(t-\tau, 1)+k_{L} c_{1}\left(k_{0} \tilde{Q}(t-\tau)\right. \\
& \left.+\tilde{Q}\left(t-\frac{2}{\lambda_{2}}-\frac{2}{\lambda_{3}}\right)\right) \\
\tau_{h r} \tilde{\tilde{Q}}(t)= & -\tilde{Q}(t)+c_{2}\left(\tilde{\alpha}_{1}(t, 1)-\tilde{\alpha}_{2}(t, 1)\right) \\
& +2 P_{0}(\tilde{y}(t)) .
\end{aligned}
$$

Using this representation, we can write the following lemma:

Lemma 1: Consider the error system (25)-(29). If $\tilde{Q}$ converges to zero, then the states $\tilde{\alpha}(t, z)$ and $\tilde{\beta}(t, z)$ also converge to zero.

Proof: If $\tilde{Q}(t)$ converges to zero, then, from the fact that $\left|k_{0} k_{L}\right|<1$, we have that $\tilde{\beta}_{2}(t, 0)$ converges to zero. Similar results hold for $\tilde{\alpha}_{1}(t, 1)$ and $\tilde{\alpha}_{2}(t, 1)$. Then, using the transport structure of (25)-(29), it is straightforward to prove the convergence to zero of the states $\tilde{\alpha}(t, z)$ and $\tilde{\beta}(t, z)$.

Using Lemma 1, we only need to focus on the state $\tilde{Q}$. We now use the solution (30)-(34) to rewrite the model of $\tilde{Q}$ as a neutral system. This allows us to find $P_{0}$ guaranteeing the convergence of $\tilde{Q}$ to its zero-equilibrium.

Denoting by $s$ the Laplace variable and taking the Laplace transform of (30)-(34), we obtain

$$
\begin{aligned}
2 \tilde{y}(s) & =\left(1+k_{L} \mathrm{e}^{-\frac{2}{\lambda_{3}} s}\right) \tilde{\beta}_{2}(s, 0) \\
\left(1-k_{0} k_{L} \mathrm{e}^{-\tau s}\right) \tilde{\beta}_{2}(s, 0)= & c_{1} \mathrm{e}^{-\frac{1}{\lambda_{2}} s}\left(1+k_{0} \mathrm{e}^{-\frac{2}{\lambda_{1}} s}\right) \tilde{Q}(s) \\
\left(1-k_{0} k_{L} \mathrm{e}^{-\tau s}\right) \tilde{\alpha}_{1}(s, 1)= & k_{0} c_{1}\left(\mathrm{e}^{-\frac{2}{\lambda_{1}} s}+k_{L} \mathrm{e}^{-\tau s}\right) \tilde{Q}(s) \\
\left(1-k_{0} k_{L} \mathrm{e}^{-\tau s}\right) \tilde{\alpha}_{2}(s, 1)= & k_{L} c_{1}\left(k_{0} \mathrm{e}^{-\tau s}+\mathrm{e}^{-\frac{2}{\lambda_{2}} s-\frac{2}{\lambda_{3}} s}\right) \tilde{Q}(s), \\
\left(s \tau_{h r}+1\right) \tilde{Q}(s)= & c_{2}\left(\tilde{\alpha}_{1}(s, 1)-\tilde{\alpha}_{2}(s, 1)\right) \\
& +2 P_{0}(\tilde{y}(s)) .
\end{aligned}
$$

Multiplying (39) by $\left(1-k_{0} k_{L} \mathrm{e}^{-\tau s}\right)$ and substituting (37)(38) in the resulting expression we obtain

$$
\begin{gathered}
\left(1-k_{0} k_{L} \mathrm{e}^{-\tau s}\right)\left(s \tau_{h r}+1\right) \tilde{Q}(s)=c_{2} c_{1}\left(k_{0} \mathrm{e}^{-\frac{2}{\lambda_{1}} s}\right. \\
\left.-k_{L} \mathrm{e}^{-\frac{2}{\lambda_{2}} s-\frac{2}{\lambda_{3}} s}\right) \tilde{Q}(s)+\left(1-k_{0} k_{L} \mathrm{e}^{-\tau s}\right) 2 P_{0}(\tilde{y}(s)) .
\end{gathered}
$$

Besides, multiplying (35) by $\left(1-k_{0} k_{L} \mathrm{e}^{-\tau s}\right)$ and substituting (36), we have that

$$
\begin{aligned}
2\left(1-k_{0} k_{L} \mathrm{e}^{-\tau s}\right) \tilde{y}(s)= & c_{1} \mathrm{e}^{-\frac{1}{\lambda_{2}} s}\left(1+k_{L} \mathrm{e}^{-\frac{2}{\lambda_{3}} s}\right) \\
& \times\left(1+k_{0} \mathrm{e}^{-\frac{2}{\lambda_{1}} s}\right) \tilde{Q}(s) .
\end{aligned}
$$




\section{Design of the operator $P_{0}$}

We are now able to define the operator $P_{0}$ such that $\tilde{Q}$ converges to zero.

Theorem 2: Let us define the operator $P_{0}(\tilde{y}(t))$ for $t \geq$ $\frac{4}{\lambda_{1}}-\frac{1}{\lambda_{2}}-\frac{2}{\lambda_{3}}$ by

$$
\begin{aligned}
P_{0}(\tilde{y}(t))= & -k_{0} P_{0}\left(\tilde{y}\left(t-\frac{2}{\lambda_{1}}\right)\right)-k_{L} P_{0}\left(\tilde{y}\left(t-\frac{2}{\lambda_{3}}\right)\right) \\
& -k_{0} k_{L} P_{0}\left(\tilde{y}\left(t-\frac{2}{\lambda_{1}}-\frac{2}{\lambda_{3}}\right)\right) \\
& +2 c_{2} k_{L} \tilde{y}\left(t-\frac{2}{\lambda_{3}}-\frac{1}{\lambda_{2}}\right) \\
& -2 c_{2} k_{L} k_{0}^{2} \tilde{y}\left(t-\frac{4}{\lambda_{1}}-\frac{1}{\lambda_{2}}-\frac{2}{\lambda_{3}}\right)
\end{aligned}
$$

and $P_{0}(\tilde{y}(t))=0$ otherwise. Then, the state $\tilde{Q}$, that satisfies the delayed differential equation (40), exponentially converges to zero. Consequently, the observer system (20)(24) converges to the real system (12)-(16).

Proof: Let us first rewrite the operator $P_{0}$ in the Laplace domain. Applying the Laplace transform into (42), we have

$$
\begin{aligned}
& 2\left(1+k_{L} \mathrm{e}^{-\frac{2}{\lambda_{3}} s}\right)\left(1+k_{0} \mathrm{e}^{-\frac{2}{\lambda_{1}} s}\right) P_{0}(\tilde{y}(s))= \\
& \left(c_{2} k_{L} \mathrm{e}^{-\frac{1}{\lambda_{2}} s-\frac{2}{\lambda_{3}} s}-c_{2} k_{0}^{2} k_{L} \mathrm{e}^{-\frac{2}{\lambda_{1}} s} \mathrm{e}^{\frac{1}{\lambda_{2}} s} \mathrm{e}^{-\tau s}\right) 2 \tilde{y}(s) .
\end{aligned}
$$

Due to Condition 1, the function $\left(1+k_{L} \mathrm{e}^{-\frac{2}{\lambda_{3}} s}\right)(1+$ $\left.k_{0} \mathrm{e}^{-\frac{2}{\lambda_{1}} s}\right)$ is strictly positive in the complex right half plane. Then, the operator $P_{0}$ is properly defined. Consequently, we have $P_{0}(\tilde{y}(s))=K(s) \tilde{y}(s)$, where $K(s)$ is defined by

$$
K(s)=\frac{c_{2} k_{L} \mathrm{e}^{-\frac{1}{\lambda_{2}} s-\frac{2}{\lambda_{3}} s}-c_{2} k_{0}^{2} k_{L} \mathrm{e}^{-\frac{2}{\lambda_{1}} s} \mathrm{e}^{\frac{1}{\lambda_{2}} s} \mathrm{e}^{-\tau s}}{\left(1+k_{L} \mathrm{e}^{-\frac{2}{\lambda_{3}} s}\right)\left(1+k_{0} \mathrm{e}^{-\frac{2}{\lambda_{1}} s}\right)} .
$$

Therefore,

$$
\left(1-k_{0} k_{L} \mathrm{e}^{-\tau s}\right) P_{0}(\tilde{y}(s))=\left(1-k_{0} k_{L} \mathrm{e}^{-\tau s}\right) K(s) \tilde{y}(s) .
$$

Using (41) and the commutative property of the operators implies that

$$
\begin{aligned}
2\left(1-k_{0} k_{L} \mathrm{e}^{-\tau s}\right) P_{0}(\tilde{y}(s))= & c_{1}\left(c_{2} k_{L} \mathrm{e}^{-\frac{2}{\lambda_{2}} s-\frac{2}{\lambda_{3}} s}\right. \\
& \left.-c_{2} k_{0}^{2} k_{L} \mathrm{e}^{-\frac{2}{\lambda_{1}} s} \mathrm{e}^{-\tau s}\right) \tilde{Q}(s),
\end{aligned}
$$

and using (40), we obtain

$$
\begin{aligned}
\left(1-k_{0} k_{L} \mathrm{e}^{-\tau s}\right)\left(s \tau_{h r}+1\right) \tilde{Q}(s)= & c_{2} c_{1}\left(1-k_{0} k_{L} \mathrm{e}^{-\tau s}\right) \\
& \times k_{0} \mathrm{e}^{-\frac{2}{\lambda_{1}} s} \tilde{Q}(s) .
\end{aligned}
$$

Due to the fact that the operator $\left(1-k_{0} k_{L} \mathrm{e}^{-\tau s}\right)$ does not vanish on the right half plane, we can define $\tilde{Q}_{1}=(1-$ $\left.k_{0} k_{L} \mathrm{e}^{-\tau s}\right) \tilde{Q}(s)$. We then have the detectability of $\tilde{Q}$ from the new variable $\tilde{Q}_{1}$ (i.e. if $\tilde{Q}_{1}$ converges to zero, so does $\tilde{Q})$. This yields

$$
\left(s \tau_{h r}+1\right) \tilde{Q}_{1}(s)=c_{2} c_{1} k_{0} \mathrm{e}^{-\frac{2}{\lambda_{1}} s} \tilde{Q}_{1}(s) .
$$

This equation rewrites as follows in the temporal domain

$$
\dot{\tilde{Q}}_{1}(t)=-\frac{1}{\tau_{h r}} \tilde{Q}_{1}(t)+\frac{c_{2} c_{1} k_{0}}{\tau_{h r}} \tilde{Q}_{1}\left(t-\frac{2}{\lambda_{1}}\right) .
$$

It corresponds exactly to (1) with $a=\frac{1}{\tau_{h r}}, b=-\frac{c_{2} c_{1} k_{0}}{\tau_{h r}}$ and $\tau_{0}=\frac{2}{\lambda_{1}}$. Using Condition 2, we have $b>|a|$ and $\tau_{0}<\frac{\arccos \left(-\frac{a}{b}\right)}{\sqrt{b^{2}-a^{2}}}$. Thus, using Theorem 1 , we have that $\tilde{Q}_{1}$ converges to zero. This implies the convergence of $\tilde{Q}$ to zero because of the detectability of $\tilde{Q}$ from $\tilde{Q}_{1}$. Finally, Lemma 1 implies the convergence of the error system to zero. This concludes the proof.

\section{Simulation Results}

In this section, we present the simulation results of the proposed observer design. The numerical tests were evaluated using the model (12)-(16) as a virtual plant and both observers design, i.e., the system (20)-(24) with $P_{0}$ computed by (42). The numerical values of the plant were $x_{0}=\frac{L}{4}$ $\mathrm{m}, x_{m}=\frac{1.25 L}{4} \mathrm{~m}, L=1.4 \mathrm{~m}, \lambda_{1}=975.9 \mathrm{~s}^{-1}, \lambda_{2}=$ $3903.6 \mathrm{~s}^{-1}, \lambda_{3}=354.87 \mathrm{~s}^{-1}, c_{1}=2.31 \mathrm{~s} \mathrm{~m}^{-3}, c_{2}=$ $1.01 \mathrm{~W} \mathrm{~Pa}^{-1}, \tau_{h r}=0.03 \mathrm{~s}, k_{0}=k_{L}=-0.93$. The observer was initialized at zero whereas the real system was initialized at $Q(0)=0.2, R_{1}(0, x)=20.4939 \cos (\pi x)+0.5 \sin (\pi x)$, $R_{2}(0, x)=-20.4939 \cos (\pi x)+0.5 \sin (\pi x)$ (the values of $\alpha_{i}$ and $\beta_{i}$ at $t=0$ can then be obtained from (9)-(10)). Simulations were carried out with Simulink.

Figure 3 shows the simulation results of the observer design with the linear operator $P_{0}$ computed by (42). Due to the delayed expressions in (42), $P_{0}(t)$ is null for values of $t \leq \frac{2}{\lambda_{2}}$ (see the bottom graphic of Figure 3). After the initial transient, the output error dynamics asymptotically converges to zero as time goes to infinity, as can be seen in the top graphic of Figure 3.

\section{CONCLUSION}

In this paper, we present a state observer design to estimate the linearized thermoacoustic dynamics of a Rijke tube. The approach is based on a Luenberger-type structure and assumes in-domain acoustic pressure measurements. As a first step in our design, the system equations were rewritten in the form of a delayed differential equation. Then, the observer was imposed as a copy of these equations plus an output injection term, which in this case is a linear operator. Using some results from the literature, we found the expression of this operator that guarantee the exponential convergence of the estimation error where the exponential convergence rate given by the physical parameters of the system. Simulation results show the performance of the proposed design.

In future works, experiments will be carried out to determine the suitability of this observer in a real setting. In this case, the robustness properties of the proposal must be studied since some parameters, such as the heat release time constant, are roughly known in practice and influence the convergence rate. In addition, the performance of the proposed observer with the backstepping control law developed in [5] will be analyzed. Other directions include the extension of the observer for the case of several acoustic pressure measurements along the domain.

\section{REFERENCES}

[1] J. Auriol and F. Di Meglio. Robust output feedback stabilization for two heterodirectional linear coupled hyperbolic PDEs. Automatica (a), 2019

[2] N. Christian, A. Wilhelmsen, and F. Di Meglio. An observer for the electrically heated vertical Rijke tube with nonlinear heat release an linearized acoustics (to appear). In 2020 IFAC World Congress, 2020.

[3] G. A. de Andrade and R. Vazquez. A backstepping-based observer for estimation of thermoacoustic oscillations in a rijke tube with indomain measurements (to appear). In 2020 IFAC World Congress, 2020. 
[4] G. A. de Andrade, R. Vazquez, and D. J. Pagano. Backstepping-based linear boundary observer for estimation of thermoacoustic instabilities in a rijke tube. In 2018 IEEE Conference on Decision and Control $(C D C)$, pages 2164-2169. IEEE, 2018.

[5] G. A. de Andrade, R. Vazquez, and D. J. Pagano. Backstepping stabilization of a linearized ODE-PDE rijke tube model. Automatica, 96:98-109, 2018.

[6] J. P. Epperlein, B. Bamieh, and K. J. Astrom. Thermoacoustics and the rijke tube: Experiments, identification, and modeling. IEEE Control Systems Magazine, 35(2):57-77, 2015.

[7] M. A. Heckl. Active control of the noise from a Rijke tube. Journal of Sound and Vibration, 124(1):117-133, 1988.

[8] S. Illingworth and A. S. Morgans. Adaptive feedback control of combustion instability in annular combustors. Combustion Science and Technology, 182(2):143-164, 2010.

[9] X. Li and D. Zhao. Feedback control of self-sustained nonlinear combustion oscillations. Journal of Engineering for Gas Turbines and Power, 138(6):061505, 2015.

[10] T. C. Lieuwen and V. Yang. Combustion instabilities in gas turbine engines: Opeartional experience, fundamental mechanisms, and modeling. AIAA, 2005.

[11] G. P. Liu and S. Daley. Output-model-based predictive control of unstable combustion systems using neural networks. Control Engineering Practice, 7(5):591-600, 1999.

[12] S.-I. Niculescu. Delay effects on stability: a robust control approach, volume 269. Springer Science \& Business Media, 2001.

[13] E. A. Powell and B. T. Zinn. Application of the Galerkin method in the solution of combustion instability problems. In Proceedings of XIX Congress of the International Astronautical Federation, 1968.

[14] R. L. Raun, M. W. Beckstead, J. C. Finlinson, and K. P. Brooks. A review of Rijke tubes, Rijke burners and related devices. Progress in Energy and Combustion Science, 19(4):313 - 364, 1993.

[15] P. L. Rijke. Notice of a new method of causing a vibration of the air contained in a tube open at both ends. Philosophical Magazine 4, 17(116):419-422, 1859

[16] J. Rubio-Hervas, M. Reyhanoglu, and E. Mackunis. Observer-based sliding mode control of Rijke-type combustion instability. Journal of low frequency noise, vibration and active control, 34(2):201-217, 2015.

[17] U. Zalluhoglu, A. S. Kammer, and N. Olgac. Delayed feedback control laws for Rijke tube thermoacoustic instability, synthesis, and experimental validation. IEEE Transactions on Control Systems Technology, 24(5):1861 - 1868, 2016.

[18] M. Zhang, J. Li, W. Cheng, and T. Li. Active control of thermoacoustic instability using microsecond plasma discharge. Journal of Applied Physics, 127:033301, 2020.

[19] D. Zhao, Z. Lu, H. Zhao, X. Y. Li, B. Wang, and P. Liu. A review of active control approaches in stabilizingcombustion systems in aerospace industry. Progress in Aerospace Sciences, 97:35-60, 2018
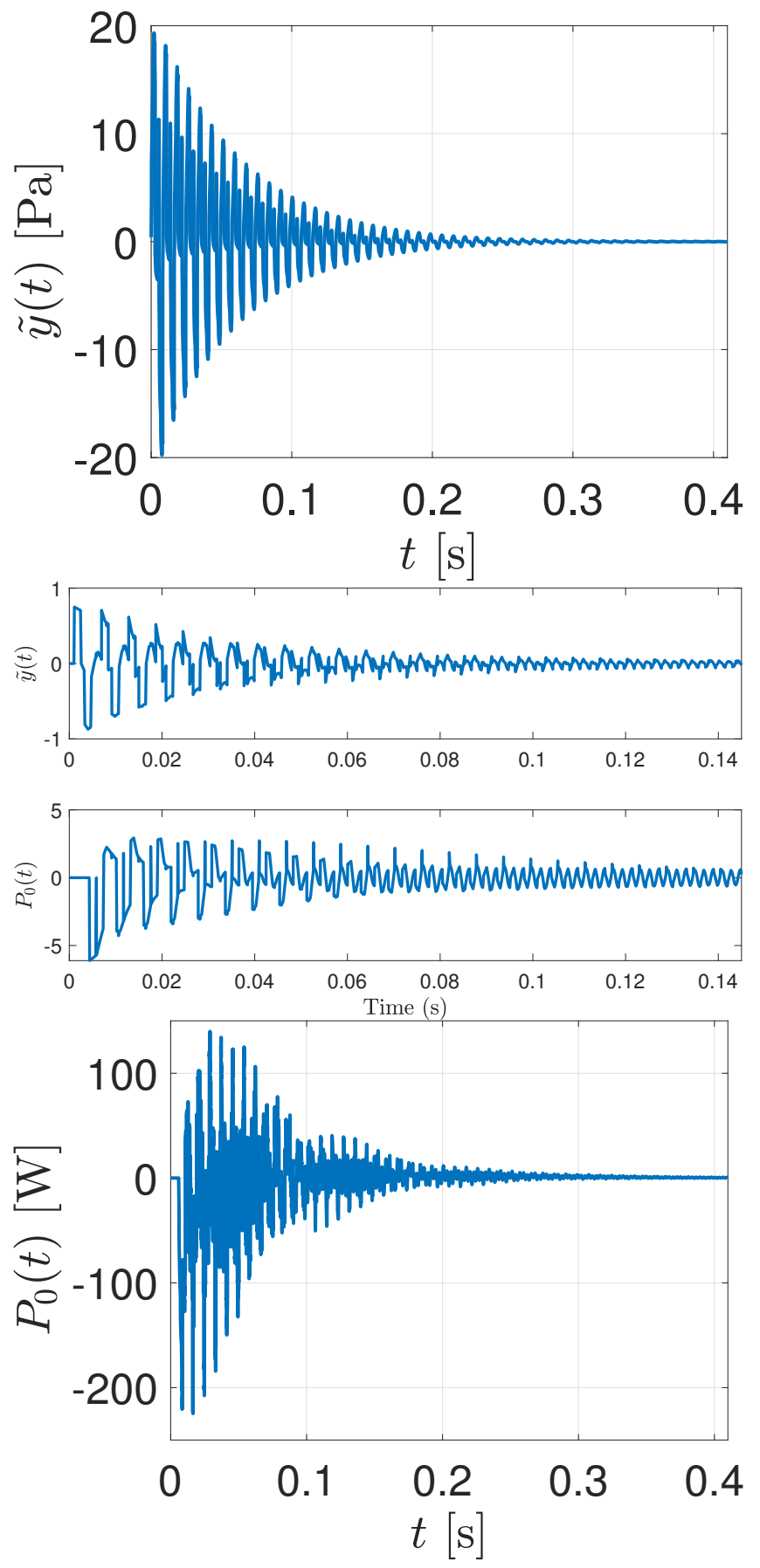

Fig. 3. Output error dynamics $\tilde{y}$ (top plot), $\tilde{Q}$ (middle plot), and $P_{0}$ evolution (bottom plot) as a function of time. 